\title{
A TIME-DEPENDENT INTERFACE PROBLEM FOR TWO-DIMENSIONAL EDDY CURRENTS*
}

\author{
BY \\ R. C. MACCAMY ${ }^{1}$ (Carnegie-Mellon University) \\ AND \\ MANIL SURI ${ }^{1}$ (University of Maryland, Baltimore County)
}

1. Introduction. This paper concerns what we term a parabolic-elliptic interface problem in the plane. It arises in the study of two-dimensional eddy-current problems in electromagnetic theory. The physical situation is discussed in Sec. 7.

Let $\Gamma$ be a smooth, simple, closed curve in the plane with interior $\Omega^{-}$and exterior $\Omega^{+}$. Let $\alpha$ be a smooth, positive function in $\bar{\Omega}^{-}$and $\mathbf{K}$ a smooth field of symmetric, positive definite linear transformations in $\bar{\Omega}^{-}$. We denote by $L$ the parabolic operator

$$
L u=\alpha \dot{u}-\operatorname{div}(\mathbf{K} \operatorname{grad} u),
$$

where $\dot{u}$ indicates differentiation with respect to $t$.

We let $T>0$ be fixed, and for any set $R \subset E_{2}$ we write $R_{T}$ for $R \times(0, T)$. For any $f$ on $E_{T}^{2}$ we write $f^{ \pm}$for its limits on $\Gamma_{T}$ from $\Omega_{T}^{ \pm}$. If $\mathbf{n}$ is the outer normal to $\Gamma$, we denote by $u_{n}$ and $W$ the operators

$$
u_{n}=\operatorname{grad} u \cdot \mathbf{n}, \quad W u=(\mathbf{K} \operatorname{grad} u) \cdot \mathbf{n} .
$$

Suppose we are given smooth functions $F$ on $\bar{\Omega}_{T}^{-}, f$ and $g$ on $\bar{\Gamma}_{T}$, and $A$ on $[0, T]$. Then consider the following:

Parabolic-Elliptic Problem (PEP). Find $u$ such that

$$
\begin{gathered}
L u=F \quad \text { in } \Omega_{T}^{-} ; \quad \Delta u=0 \quad \text { in } \Omega_{T}^{+}, \\
u(x, 0)=0 \quad \text { in } \Omega^{-}, \\
u^{-}=u^{+}+f, \quad W u^{-}=u_{n}^{+}+g \quad \text { on } \Gamma_{T}, \\
u(t, x)-A(t) \log |x|=O(1) \quad \text { as }|x| \rightarrow \infty .
\end{gathered}
$$

We will not state precise smoothness conditions except to say that (1.3) should hold pointwise and Green's theorem should be applicable. The latter yields the following elementary result proved at the end of the section.

\footnotetext{
${ }^{*}$ Received April 12, 1985.

${ }^{1}$ This work was supported by the National Science Foundation under Grant DMS- 8400986.
} 
THEOREM 1.1.(PEP) has at most one solution.

We show in Sec. 7 that the eddy-current problem leads to the following problem.

Parabolic-Hyperbolic Problem (PHP). Find $u$ such that

$$
\begin{gathered}
L u=F \text { in } \Omega_{T}^{-} ; \quad \Delta u-\beta^{2} \ddot{u}=0 \quad \text { in } \Omega_{T}^{+}, \\
u(x, 0)=0 \quad \text { in } \Omega^{-} ; \quad u(x, 0)=\dot{u}(x, 0)=0 \quad \text { in } \Omega^{+}, \\
u^{-}=u^{+}+f, \quad W u^{-}=u_{n}^{+}+g \quad \text { on } \Gamma_{T} .
\end{gathered}
$$

We do not, as yet, know how to treat (1.4). For a large class of problems, however, the parameter $\beta^{2}$ is very small, and it is common practice to take it equal to zero (the quasistatic approximation). Thus we are led to (PEP). The extra condition $(1.3)_{4}$ is a technical consequence of the approximation (see Sec. 7). It causes considerable mathematical complication but is essential if the problem is to be physically meaningful.

In the eddy current application we will have

$$
\int_{\Gamma} g d s=0
$$

and we assume from now on that this condition holds.

In Sec. 2 we will convert (PEP) to a problem (NLP) in which

$$
L u=F \quad \text { in } \Omega_{T}^{-} ; \quad u(x, 0)=0 \quad \text { in } \Omega^{-},
$$

but we have a nonlocal boundary condition on $\Gamma_{T}$. In Sec. 3 we give a variational formulation (VP) for (NLP). We also present a Galerkin procedure for (VP), consisting of a family of approximate problems $(\mathrm{AVP})_{h}$ depending on a parameter $h$. We have two main results:

1. The problems (AVP) ${ }_{h}$ have solutions that converge to a (unique) generalized solution of (VP).

2. The convergence is optimal.

These results are stated precisely in Sec. 3 and the proofs are presented in Secs. 4 and 5. Sec. 6 contains some remarks on numerical implementation. Many of the ideas here come from [1] and [7].

Proof of Theorem 1.1. It suffices to show that the only solution for $F, f, g$, and $A$ all identically zero is $u \equiv 0$. Suppose $u$ is such a solution. We multiply the differential equation in $\Omega_{T}^{-}$by $u$ and integrate over $\Omega_{t}^{-}$. Green's theorem yields

$$
\begin{aligned}
& \frac{\alpha}{2} \int_{\Omega} u(x, t)^{2} d x+\int_{0}^{t} \int_{\Omega} \mathbf{K}(x) \operatorname{grad} u(x, \tau) \cdot \operatorname{grad} u(x, \tau) d x d \tau \\
& \quad-\int_{0}^{t} \int_{\Gamma} W u(x, \tau)^{-} u(x, \tau)^{-} d x d \tau=0 .
\end{aligned}
$$

Since $u$ is bounded as $|x| \rightarrow \infty$ and $\Delta u=0$ in $\Omega_{T}^{+}$, we have $\nabla u=0\left(|x|^{-2}\right)$. We can perform a similar integration outside (with a limiting argument), obtaining

$$
\int_{0}^{t} \int_{\Omega^{+}}|\operatorname{grad} u(x, \tau)|^{2} d x d \tau+\int_{0}^{t} \int_{\Gamma} u_{n}^{+}(x, \tau) u(x, \tau)^{+} d x d \tau=0
$$

We add the results and use $(1.3)_{2}$ and $(1.3)_{3}$ to conclude that $u(x, t) \equiv 0$ in $\Omega^{-}$, $\operatorname{grad} u(x, t) \equiv 0$ in $\Omega^{+}$, and $u^{+}(x, t) \equiv u(x, t)=0$ on $\Gamma$, so $u(x, t) \equiv 0$ in $\Omega^{+}$. 
2. The nonlocal boundary problem. We need some results from potential theory. Let $g(x, y)=(2 \pi)^{-1} \log |x-y|$ and let $\mathscr{S}$ and $\mathscr{D}$ denote the simple and double layers:

$$
\begin{gathered}
\mathscr{S}[\varphi](x)=\int_{\Gamma} \varphi(y) g(x, y) d s_{y} ; \\
\mathscr{D}[\varphi](x)=\int_{\Gamma} \varphi(y) \frac{\partial}{\partial n_{y}} g(x, y) d s_{y} .
\end{gathered}
$$

For smooth $\Gamma$ and $\varphi$, the following results are well known:

$$
\begin{gathered}
\Delta \mathscr{S}[\varphi]=\Delta \mathscr{D}[\varphi]=0 \text { in } \Omega^{ \pm}, \\
\mathscr{S}[\varphi]^{+}=\mathscr{S}[\varphi]^{-}=S[\phi] \text { on } \Gamma, \\
(\mathscr{D}[\varphi])^{ \pm}=\mp \frac{1}{2} \varphi+D[\phi] \quad \text { on } \Gamma, \\
{[\partial \mathscr{S}[\varphi] / \partial n]^{ \pm}= \pm \frac{1}{2} \varphi+N[\varphi] \quad \text { on } \Gamma,} \\
\mathscr{S}[\varphi]=m[\varphi] \log |x|+O\left(|x|^{-1}\right), \quad m[\varphi]=(2 \pi)^{-1} \int_{\Gamma} \varphi(y) d s_{y} \quad \text { as }|x| \rightarrow \infty
\end{gathered}
$$

Here $S$ is an integral operator with logarithmic kernel, while $D$ and $N$ are integral operators with smooth kernels.

Suppose now that $u$ is a solution of (PEP). Then $(1.3)_{4}$ can be sharpened to

$$
D^{\alpha}\{u(x, t)-A \log |x|-B(t)\}=O\left(|x|^{-|\alpha|-1}\right) \text { as }|x| \rightarrow \infty
$$

for some function $B$ and for any spatial derivative $D^{\alpha}$. Then one can apply Green's theorem to obtain the representation

$$
u=\mathscr{S}\left[u_{n}^{+}\right]-\mathscr{D}\left[u^{+}\right]+C, \quad C=-B \text { in } \Omega_{T}^{+} .
$$

Eqs. (2.3) and (2.2) 1,2 yield

$$
\frac{1}{2} u^{+}=S\left[u_{n}^{+}\right]-D\left[u^{+}\right]+C \text { on } \Gamma_{T} .
$$

Now we use the interface conditions. We have $u^{+}=u^{-}-f$, and if we put $(W u)^{-}=\phi$, then $u_{n}^{+}=\phi-g$. We substitute into (2.4) to obtain

$$
\frac{1}{2} u^{-}+D\left[u^{-}\right]-S[\phi]-C=D[f]-S[g]+\frac{1}{2} f \equiv G \quad \text { on } \Gamma_{T} .
$$

From (2.3), (1.3) 4 , and (2.2) 5 we obtain, by (1.5),

$$
m[\phi]=A \text {. }
$$

The formulas above lead us to the following:

Nonlocal Problem (NLP). Find $\{u, \varphi, C\}$ such that

$$
\begin{gathered}
L u=F \text { in } \Omega_{T}^{-} ; \quad u(x, 0)=0 \quad \text { in } \Omega^{-}, \\
W u^{-}=\phi \quad \text { on } \Gamma_{T}, \\
\frac{1}{2} u^{-}+D\left[u^{-}\right]-S[\phi]-C=G \quad \text { on } \Gamma_{T}, \\
m[\phi]=A .
\end{gathered}
$$


TheOrem 2.1. Suppose $\{u, \varphi, C\}$ is a solution of (NLP), and we set

$$
u=\mathscr{S}[\phi-g]-\mathscr{D}\left[u^{-\cdots}-f\right]+C \text { in } \Omega_{T}^{+} .
$$

Then $u$ is a solution of (PEP) with $\phi=(W u)^{-}$.

Proof. Let $u$ denote the right side of (2.8). We have $\Delta u=0$ in $\Omega_{T}^{+}$, while (2.6) and (2.2) show that $(1.3)_{4}$ is satisfied. By $(2.2)_{2},(2.2)_{3}$, and $(2.5)$, we have $u^{+}=u^{-}-f$. Now consider $u$ in $\Omega_{T}^{-}$. By $(2.2)_{2},(2.2)_{3}$, and (2.4) we have

$$
u^{-}=S[\phi-g]+\frac{1}{2}\left(u^{-}-f\right)-D\left[u^{-}-f\right]+C=0 .
$$

We conclude that $u \equiv 0$ in $\Omega_{T}^{-}$. But then $(2.2)_{4}$ yields $\phi-g=u_{n}^{+}$.

REMAKK. (NLP) is an extension of an idea used in [5] for static problems and in [1] for time-periodic ones.

3. The variational problem. Eq. (2.7) and an application of the divergence theorem yield, for an arbitrary function $v$,

$$
\int_{\Omega} \alpha \dot{u} v d x+\int_{\Omega} \mathbf{K} \operatorname{grad} u \cdot \operatorname{grad} v d x-\int_{\Gamma} v \phi d s=\int_{\Omega} F v d x .
$$

Similarly we multiply $(2.7)_{3}$ by a test function $\psi$ and integrate over $\Gamma$ to obtain

$$
\int_{\Gamma} u^{-} \psi d s+2 \int_{\Gamma} D\left[u^{-}\right] \psi d s-2 \int_{\Gamma} S[\phi] \psi d s-4 \pi C m[\psi]=2 \int_{\Gamma} G \psi d s .
$$

Finally $(2.9)_{4}$ yields, for any constant $k$,

$$
4 \pi k m[\phi]=4 \pi A k .
$$

Let us introduce the following notation:

$$
\begin{aligned}
& a(\{u, \varphi, C\},\{v, \psi, k\})= \int_{\Omega} \mathbf{K} \operatorname{grad} u \cdot \operatorname{grad} v d x-\int_{\Gamma} v^{-} \phi d s+\int_{\Omega} u^{-} \psi d s \\
& \quad+2 \int_{\Gamma} D\left[u^{-}\right] \psi d s-2 \int_{\Gamma} S[\phi] \psi d s-4 \pi C m[\psi]+4 \pi k m[\phi], \\
&\left.\mathscr{F}_{(F, G, A)}(\{v, \psi, k\})=\int_{\Gamma} F v d x+2 \int_{\Gamma} G \psi d s+4 \pi A k .4\right)
\end{aligned}
$$

Then (3.1)-(3.3) give our variational formulation.

Variational Problem $(V P)$. Given $\{F, G, A\}$ on $[0, T)$, find $\{u, \varphi, C\}$ on $[0, T)$ such that $u(x, 0)=0$ and for any $\{v, \psi, k\}$,

$$
\int_{\Omega} \alpha \dot{u} v d x+a(\{u, \varphi, C\},\{v, \psi, k\})=\widetilde{\mathscr{F}}_{(F, G, A)}(\{v, \psi, k\}) .
$$

The following result is easily verified.

THEOREM 3.1. If $\{u, \varphi, C\}$ is a solution of (VP) with sufficient smoothness, then $\{u, \varphi, C\}$ is a solution of (NLP).

We also consider approximate problems. Let $\left\{H^{h}\right\}$ and $\left\{B^{h}\right\}$ be families of finite-dimensional spaces depending on a parameter $h>0$. Pui $\mathscr{H}^{h}=H^{h} \times B^{h} \times \mathbf{R}$. 
Approximate Variational Problems $(A V P)_{h}$. Given $\{F, G, A\}$ on $[0, T)$, find $\left\{u^{h}, \varphi^{h}, C^{h}\right\} \in \mathscr{H}^{h}$ on $[0, T)$ such that $u^{h}(x, 0)=0$ and for any $\left\{v^{h}, \psi^{h}, k\right\} \in \mathscr{H}^{h}$,

$$
\int_{\Omega} \alpha \dot{u}^{h} v^{h} d x+a\left(\left\{u^{h}, \varphi^{h}, C^{h}\right\},\left\{v^{h}, \psi^{h}, k\right\}\right)=\mathscr{F}_{(F, G, A)}\left(\left\{v^{h}, \psi^{h}, k\right\}\right) .
$$

We will obtain generalized solutions of (VP), and we formulate this concept now. We will require of $u$ and $v$ that they lie in $H_{1}(\Omega)$ so that $u^{-}, v^{-}$lie in $H_{1 / 2}(\Gamma)$. Since $\varphi$ is to be a conormal derivative we expect that $\varphi$, and hence $\psi$, should be in $H_{-1 / 2}(\Gamma)$. We put

$$
H=H_{1}(\Omega), \quad B=H_{-1 / 2}(\Gamma)
$$

and let $H^{\prime}$ and $B^{\prime}$ be their duals with respect to $L_{2}(\Omega)$ and $L_{2}(\Gamma)$. Thus $B^{\prime}=H_{1 / 2}(\Gamma)$. Naturally $H^{h}$ and $B^{h}$ are to be subspaces of $H$ and $B$. We put

$$
\mathscr{H}=H \times B \times \mathbf{R}, \quad \mathscr{H}^{\prime}=H^{\prime} \times B^{\prime} \times \mathbf{R} .
$$

We can extend $a$ and $\mathscr{F}_{(F, G, A)}$ to $\mathscr{H} \times \mathscr{H}$ and $\mathscr{H}$, respectively. To do this, we need to interpret some of the integrals as duality pairings. For any space $J$ we write $\langle\tau, v\rangle$ for such a pairing of $\tau \in J^{\prime}, v \in J$. Then we replace $\int_{\Gamma} v^{-} \varphi d s$ and $\int_{\Gamma} u^{-} \psi d s$ by $\left\langle v^{-}, \varphi\right\rangle$ and $\left\langle u^{-}, \psi\right\rangle$. For $\dot{u}$ and $F$ in $H^{\prime}$, we replace $\int_{\Omega} \alpha \dot{u} v d x$ and $\int_{\Omega} F v d x$ by $\langle\alpha \dot{u}, v\rangle$ and $\langle F, v\rangle$. $m[\varphi]$ and $m[\psi]$ can be interpreted as duality pairings with the function which is identically one. For the remaining terms we need the following result from [4].

Lemma 3.1. For any $r \geqslant-1 / 2, S$ maps $H_{r}(\Gamma)$ into $H_{r+1}(\Gamma)$ and $D$ maps $H_{r}(\Gamma)$ into $H_{r+2}(\Gamma)$.

Lemma (3.1) shows that we may replace $2 \int_{\Gamma} D\left[u^{-}\right] \psi d s-2 \int_{\Gamma} S[\phi] \psi d s$ by $2\left\langle D\left[u^{-}\right], \psi\right\rangle$ $-2\langle S[\phi], \psi\rangle$. Moreover, the lemma and (2.8) show that if $f \in B^{\prime}$ and $g \in B$ then $G \in B^{\prime}$ and we can replace $2 \int_{\Gamma} G \psi d s$ by $2\langle G, \psi\rangle$. Thus we can put

$$
\begin{aligned}
a(\{u, \varphi, C\},\{v, \psi, k\})= & \int_{\Omega} K \operatorname{grad} u \cdot \operatorname{grad} v d x-\langle v, \phi\rangle+\left\langle u^{-}, \psi\right\rangle+2\left\langle D\left[u^{-}\right], \psi\right\rangle \\
& -2\langle S[\varphi], \psi\rangle-4 \pi C m[\psi]+4 \pi k m[\varphi] \mathscr{F}_{(F, G, A)}(\{v, \psi, k\}) \\
= & \langle F, v\rangle+2\langle G, \psi\rangle+4 \pi A k .
\end{aligned}
$$

Our solutions will also be generalized with respect to time. For any space $J$ we write $J_{T}$ for $L_{2}((0, T): J)$. We set

$$
\begin{aligned}
Q_{T} & =\left\{\{u, \varphi, C\}: u \in H_{T}, \varphi \in B_{T}, C \in \mathbf{R}_{T}\right\}, \\
Q_{T}^{+} & =\left\{\{u, \varphi, C\} \in Q_{T}: \dot{u} \in H_{T}^{\prime}, u(\cdot, 0)=0\right\}, \\
P_{T} & =\left\{\{F, G, A\}: F \in H_{T}^{\prime}, G \in B_{T}^{\prime}, A \in \mathbf{R}_{T}\right\} .
\end{aligned}
$$

Note that $u \in H_{T}$ and $\dot{u} \in H_{T}^{\prime}$ imply $u \in C\left([0, T]: L_{2}(\Omega)\right)$ so that $u(\cdot, 0)=0$ has meaning. The bilinear form $a$ extends to $Q_{T} \times Q_{T}$ and $\mathscr{F}_{(F, G, A)}$ to a linear functional on $Q_{T}$. Thus the following is meaningful.

Definition 3.1. For any $\{F, G, A\} \in P_{T},\{u, \varphi, C\}$ is a generalized solution of (VP) if $\{u, \varphi, C\} \in Q_{T}^{+}$and for any $\{v, \psi, k\} \in Q_{T}$,

$$
\langle\alpha \dot{u}, v\rangle+a(\{u, \varphi, C\},\{v, \psi, k\})=\mathscr{F}_{(F, G, A)}(\{v, \psi, k\}) .
$$


We will need two assumptions concerning the spaces $H^{h}$ and $B^{h}$ :

A.1. Define the $L_{2}$ projection $P^{h}: H \rightarrow H^{h}$ by $\int_{\Omega} P^{h} v w^{h} d x=\int v w^{h} d x$ for all $w^{h} \in H^{h}$. Then there is a constant $\gamma, 0<\gamma<\infty$, independent of $h$, such that

$$
\sup _{v \in H} \frac{\left\|P^{h} v\right\|_{H}}{\|v\|_{H}} \leqslant \gamma .
$$

A.2. For any $\varepsilon>0$ there is an $\bar{h}(\varepsilon)$ such that for any $h \leqslant \bar{h}(\varepsilon)$ and any $\{u, \varphi\}$ in $H_{T} \times B_{T}$ with $u_{t} \in H_{T}^{\prime}$ there is a $\left\{u^{h}, \varphi^{h}\right\} \in H_{T}^{h} \times B_{T}^{h}$ such that

$$
\left\|u-u^{h}\right\|_{H_{T}}+\left\|u_{t}-u_{t}^{h}\right\|_{H_{T}^{\prime}}+\left\|\varphi-\varphi^{h}\right\|_{B_{T}} \leqslant \varepsilon
$$

We indicate the meaning of these assumptions in Sec. 6 .

We can now state our main results under the assumptions A.1 and A.2.

TheOrem 3.2. There exists a unique generalized solution $\{u, \varphi, C\}$ of (VP). Moreover, there is a constant $K$, independent of $T$, such that

$$
\|\{u, \varphi, C\}\|_{Q_{T}^{+}} \leqslant K\|\{F, G, A\}\|_{P_{T}} .
$$

THEOREM 3.3. There exists an $\bar{h}>0$ and a $K$, independent of $T$, such that

(i) For any $h \leqslant \bar{h},(\mathrm{AVP})_{h}$ has a unique solution $\left\{u^{h}, \varphi^{h}, C^{h}\right\}$.

(ii)

$$
\left\|\{u, \varphi, C\}-\left\{u^{h}, \varphi^{h}, C^{h}\right\}\right\|_{Q_{T}^{+}} \leqslant \inf \left\|\{u, \varphi, C\}-\left\{w^{h}, \chi^{h}, l^{h}\right\}\right\|_{Q_{T}^{+}} .
$$
where $\left\{w^{h}, \chi^{h}, l^{h}\right\} \in Q_{T}^{h}=H_{T}^{h} \times B_{T}^{h} \times \mathbf{R}_{T}$.

The optimality result, Theorem 3.3(ii), yields an estimate on the order of convergence as we discuss in Sec. 6. In particular, if one uses piecewise linear finite elements for $H^{h}$ and piecewise constant elements for $B^{h}$ and if $\{u, \varphi, C\}$ has enough smoothness, then (3.14) yields $O(h)$ convergence, $h$ being a mesh size.

We expect that if one imposes enough smoothness of $f$ and $g$, then the generalized solution of (VP) will be sufficiently regular to yield a classical solution of (PEP). We have not, however, carried out the details.

4. Proof of existence. We will prove Theorem 3.2 in this section. We do so by showing that (VP) is a compact perturbation of a coercive problem and then using Riesz-Schauder theory. We let $a_{0}$ denote the bilinear form

$$
\begin{aligned}
a_{0}(\{u, \varphi, C\},\{v, \psi, k\})= & \int_{\Omega}(\mathbf{K} \operatorname{grad} u \cdot \operatorname{grad} v+u v) d x-\left\langle v^{-}, \phi\right\rangle+\left\langle u^{-}, \psi\right\rangle \\
& -2\langle S[\varphi], \psi\rangle-4 \pi C m[\psi]+4 \pi k m[\phi]
\end{aligned}
$$

on $Q_{T} \times Q_{T}$. We also let $\mathscr{R}$ and $\mathscr{J}$ be the bounded linear maps from $H_{T}$ into $H_{T}^{\prime}$ and $B_{T}^{\prime}$, defined respectively by

$$
\langle\mathscr{R} u, v\rangle=\int_{\Omega} u v d x, \quad\langle\mathscr{J} u, \psi\rangle=-2\left\langle D\left[u^{-}\right], \psi\right\rangle .
$$

To simplify the formulas, we assume $\alpha \equiv 1$. Then (3.10) becomes

$$
\langle\dot{u}, v\rangle+a_{0}(\{u, \varphi, C\},\{v, \psi, k\})=\mathscr{F}_{(F+\mathscr{Q} u, G+\mathscr{g} u, A)}(\{v, \psi, k\}) .
$$


We consider the auxiliary problem suggested by (4.3):

$$
\langle\dot{u}, v\rangle+a_{0}(\{u, \varphi, C\},\{v, \psi, k\})=\mathscr{F}_{(F, G, A)}(\{v, \psi, k\}) .
$$

The approximate problem corresponding to (4.4) is $u^{h}(x, 0)=0$, and for any $\left\{v^{h}, \psi^{h}, k\right\}$,

$$
\left\langle\dot{u}^{h}, v^{h}\right\rangle+a_{0}\left(\left\{u^{h}, \varphi^{h}, C^{h}\right\},\left\{v^{h}, \psi^{h}, k\right\}\right)=\mathscr{F}_{(F, G, A)}\left(\left\{v^{h}, \psi^{h}, k\right\}\right) .
$$

We observe that since $\mathbf{K}$ is positive definite, we have

$$
\int_{\Omega}\left(\mathbf{K} \operatorname{grad} u \cdot \operatorname{grad} u+u^{2}\right) d x \geqslant \gamma_{1}\|u\|_{H}^{2}
$$

Moreover, we have the following result, proved at the end of the section.

LEMMA 4.1. There exists a constant $\gamma_{2}>0$ such that for any $\varphi \in B$ with $m[\varphi]=0$,

$$
-\langle S[\varphi], \varphi\rangle \geqslant \gamma_{2}\|\varphi\|_{B}^{2} \text {. }
$$

Thus the form $a_{0}$ is nearly coercive. In order to exploit this coercivity, we devise a two-step procedure for solving (4.3).

We let $\overline{\mathbf{R}}$ denote the set of constant functions on $\Gamma$. We assume $B^{h} \supset \overline{\mathbf{R}}$ and for any $C \in \mathbf{R}$ let $\bar{C}$ denote the corresponding function in $\overline{\mathbf{R}}$. We have then

$$
B^{h}=\stackrel{\circ}{B}^{h} \oplus \overline{\mathbf{R}}
$$

with respect to $L_{2}(\Gamma)$, where $\AA^{h} \in \stackrel{\circ}{B}^{h}$ implies $m\left[\AA^{h}\right]=0$, and for any $\psi^{h} \in B^{h}$,

$$
\psi^{h}=\dot{\psi}^{h}+\overline{(2 \pi / l) m\left[\psi^{h}\right]},
$$

where $l$ is the length of $\Gamma$. Note that for any $C \in \mathbf{R}$,

$$
\|\bar{C}\|_{B}=|C|\|\overline{1}\|_{B} .
$$

We put $v^{h}=0, \psi^{h}=0$ in (4.5) and conclude that $m\left[\phi^{h}\right]=A$. Then (4.9) yields $\phi^{h}=\dot{\phi}^{h}+\overline{2 \pi A / l}=\dot{\phi}^{h}+\bar{\tau}$. This leads to our two-step procedure.

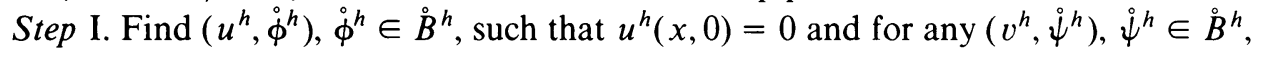

$$
\begin{aligned}
& \left\langle\dot{u}^{h}, v^{h}\right\rangle+a_{0}\left(\left\{u^{h}, \dot{\varphi}^{h}, 0\right\},\left\{v^{h}, \dot{\psi}^{h}, 0\right\}\right)=\mathscr{F}_{(\tilde{F}, \tilde{G}, 0)}\left(\left\{v^{h}, \dot{\psi}^{h}, 0\right\}\right), \\
& \tilde{F} v^{h}=\left\langle F, v^{h}\right\rangle+\left\langle\left(v^{h}\right)^{-}, \bar{\tau}\right\rangle, \quad \tilde{G} \dot{\psi}^{h}=\left\langle G, \dot{\psi}^{h}\right\rangle+2\left\langle S[\bar{\tau}], \dot{\psi}^{h}\right\rangle .
\end{aligned}
$$

Step II. Find $C^{h}$ such that for $u^{h}, \dot{\varphi}^{h}$ from Step I and any $\bar{\zeta} \in \overline{\mathbf{R}}$,

$$
\left\langle\left(u^{h}\right)^{-}, \bar{\zeta}\right\rangle-2\left\langle S\left[\dot{\varphi}^{h}\right], \bar{\zeta}\right\rangle-4 \pi C^{h} m[\bar{\zeta}]=\langle\tilde{G}, \tilde{\zeta}\rangle .
$$

One verifies that if the $\left\{u^{h}, \dot{\varphi}^{h}, C^{h}\right\}$ are determined by (4.11)-(4.13), then $\left\{u^{h}, \dot{\phi}^{h}+\bar{\tau}, C^{h}\right\}$ satisfies (4.5).

LEMMA 4.2. The problems (4.5) have unique solutions for each $h$.

Proof. We show first that (4.11) has solutions. If we choose bases for $H^{h}$ and $\stackrel{\circ}{B}^{h}$, then (4.11) will be equivalent to a system of equations of the form

$$
\begin{gathered}
\mathscr{M} \dot{\mathbf{U}}^{h}+\mathscr{A} \mathbf{U}^{h}+\mathscr{B} \dot{\Phi}^{h}=\mathscr{F}, \quad \mathbf{U}^{h}(0)=0, \\
\mathscr{S} \dot{\Phi}^{h}-\mathscr{B}^{r} \mathbf{U}^{h}=\mathscr{A} .
\end{gathered}
$$


Here $\mathbf{U}^{h}$ and $\mathscr{F}$ have values in $\mathbf{R}^{M}$, and $\Phi^{h}$ and $\mathscr{A}$ values in $\mathbf{R}^{N}$ for some $M$ and $N . \mathscr{M}$ and $\mathscr{A}$ are $M \times M$ matrices, $\mathscr{S}$ an $N \times N$ matrix, and $\mathscr{B}$ is $M \times N$. The mass matrix $\mathscr{M}$ is positive definite, and one verifies that (4.7) implies that $\mathscr{S}$ is positive definite. Accordingly one may solve (4.14) ${ }_{2}$ for $\AA^{h}$ in terms of $\mathbf{U}^{h}$ and substitute in (4.14) to obtain a differential equation for $\mathbf{U}^{h}$. Since $\mathscr{M}$ is nonsingular, this equation has a unique solution. Once $\mathbf{U}^{h}$ and $\dot{\Phi}^{h}$ are determined, (4.13) will yield $C^{h}$.

We now prove stability with respect to the data for solutions of (4.5).

LEMma 4.3. There exists a constant $K$, independent of $h$, such that the solution of (4.5) satisfies

$$
\left\|\left\{u^{h}, \phi^{h}, C^{h}\right\}\right\|_{Q_{T}} \leqslant K\|\{F, G, A\}\|_{P_{T}} .
$$

Proof. We put $v^{h}=u^{h}$ and $\psi^{h}=\dot{\phi}^{h}$ in (4.11), and we integrate from 0 to $T$. Equations (4.6) and (4.7) yield

$$
\left\|u^{h}(T)\right\|_{L_{2}(\Omega)}^{2}+\left\|u^{h}\right\|_{H_{T}}^{2}+\left\|\dot{\phi}^{h}\right\|_{B_{T}}^{2} \leqslant K_{1}\left(\|F\|_{H_{T}^{\prime}}^{2}+\|G\|_{B_{T}^{\prime}}^{2}+\|A\|_{T}^{2}\right) .
$$

Since $\left\|\left(u^{h}\right)^{-}\right\|_{B_{T}^{\prime}}$ is bounded by a constant times $\left\|u^{h}\right\|_{H_{T}}$, it will satisfy an inequality of the same type, and then (4.13) shows that the same is true for $C^{h}$. It then follows from (4.9) and the fact that $\phi^{h}=\dot{\phi}^{h}+2 \pi \bar{A} / l$ that (4.16) holds with $\phi^{h}$ replacing $\dot{\phi}^{h}$.

Now let $P^{h}$ be the $L^{2}$ projection as defined in A.1. We have

$$
\left\|\dot{u}^{h}(t)\right\|_{H^{\prime}}=\sup _{v \in H} \frac{\int_{\Omega} \dot{u}^{h}(t) v d x}{\|v\|_{H}}=\sup _{v \in H} \frac{\int_{\Omega} \dot{u}^{h}(t) P^{h} v d x}{\|v\|_{H}} .
$$

Now we use (4.5) with $v^{h}=P^{h} v, \psi^{h}=0, k=0$ to estimate the right side of this equality. If we use A.1 and (4.16), this yields

$$
\begin{aligned}
\left\|\dot{u}^{h}\right\|_{H_{T}^{\prime}}= & \sup _{v \in H}\left(1 /\|v\|_{H}\right)\left\{\left\langle F, P^{h} v\right\rangle+\left\langle\left(P^{h} v\right)^{-}, \phi^{h}\right\rangle\right. \\
& \left.-\int_{\Omega}\left(\mathbf{K} \operatorname{grad} u^{h} \cdot \operatorname{grad} p^{h} v+u^{h} P^{h} v\right) d x\right\} \\
\leqslant & K_{2}^{\prime}\left\{\|F\|_{H_{T}^{\prime}}+\left\|\phi^{h}\right\|_{B_{T}}+\left\|u^{h}\right\|_{H_{T}}\right\} \sup \frac{\left\|P^{h} v\right\|_{H}}{\left\|v^{h}\right\|_{H}} \\
\leqslant & K_{2}^{\prime}\left\{\|F\|_{H_{T}^{\prime}}+\|G\|_{B_{T}^{\prime}}^{2}+\left\|A_{T}\right\|^{2}\right\} .
\end{aligned}
$$

The estimates (4.16) and (4.17) yield (4.15).

Lemma 4.4. (i) There exists a unique solution $\{u, \varphi, C\}$ of (4.4).

(ii) There is a constant $K$ such that

$$
\|\{u, \varphi, C\}\|_{Q_{\tau}} \leqslant K\|\{F, G, A\}\|_{P_{T}} .
$$

Proof. This is a standard argument, which we only outline. From the estimate (4.15) we conclude that there is a subsequence of the $\left\{u^{h}, \varphi^{h}, C^{h}\right\}$ converging weakly to $u$, with $\left\{\dot{u}^{h}\right\}$ converging weakly to $\dot{u}$. Then one shows from A.2 that $\dot{u}$ is the generalized derivative of $u$ and that $\{u, \varphi, C\}$ satisfies (4.4). The estimate (4.18) follows from (4.15) and lower semicontinuity. 
To show that the solution of (4.4) is unique, it suffices to show that the only solution for $\{F, G, A\}=\{0,0,0\}$ is $\{0,0,0\}$. For such a solution, (4.4), with $v=0$ and $\psi=0$, yields $m[\varphi]=0$. Then we put $v=u, \psi=\varphi$, and $k=C$ in (4.4) and integrate from 0 to $T$. Equation (4.12) and Lemma 4.1 yield

$$
\|u(\cdot, T)\|_{L_{2}(\Omega)}+\|u\|_{H_{T}}+\|\varphi\|_{B_{T}}=0 .
$$

Thus $u$ and $\varphi$ are identically zero, and (4.4) yields $C=0$.

Since the solution of (4.4) is unique, it follows in the usual way that the entire Galerkin sequence $\left\{u^{h}, \varphi^{h}, C^{h}\right\}$ converges weakly to the solution $\{u, \varphi, C\}$.

We turn now to (VP). Let us first establish the uniqueness. We use a combination of the proofs of Theorems 1.1 and 2.1. Again let $\{u, \varphi, C\}$ be a solution of (3.10) for $\{F, G, A\}$ $=\{0,0,0\}$. We set $v=0$ and $\psi=0$ and deduce that $m[\varphi]=0$. Now define $u$ by

$$
u=\mathscr{S}[\varphi]-\mathscr{D}\left[u^{-}\right]+C .
$$

For $\varphi$ in $B$ or $B^{\prime},(2.2)_{1}$ is still true pointwise, as is $(2.2)_{5}$. Also $\mathscr{S}[\varphi]$ and $\mathscr{D}[\varphi]$ are in $H_{1}\left(\Omega^{-}\right) . \mathscr{D}[\varphi]$ is in $H_{1}\left(\Omega^{+}\right), \mathscr{S}[\varphi] \in H_{1}\left(\Omega^{+}\right)$if $m[\varphi]=0$, and $\mathscr{S}[\varphi]$ is always in $H_{1}^{\text {loc }}\left(\Omega^{+}\right)$(see [4]). Formulas $(2.2)_{2}-(2.2)_{4}$ continue to hold if interpreted by the trace theorem.

We take $v=0$ and $k=0$ in (3.10) and conclude that $\frac{1}{2} u^{-}+D\left[u^{-}\right]-S\left[\varphi^{-}\right]-C=0$. As in the proof of Theorem 2.1, this implies $u^{+}=u^{-}, u^{-}=0$, and $u_{n}^{+}=\varphi$. Now we proceed as in the proof of Theorem 1.1. We apply Green's theorem with a limiting argument to obtain

$$
\int_{0}^{t} \int_{\Omega^{+}}|\operatorname{grad} u|^{2} d x d \tau+\int_{0}^{t}\left\langle u^{-}, \phi\right\rangle d \tau=0
$$

On the other hand, if we take $\psi=0, k=0$, and $v=u$ in (3.10) and integrate from 0 to $t$, we obtain

$$
\frac{1}{2} \int_{\Omega^{-}} u(x, t)^{2} d x+\int_{0}^{t} \int_{\Omega^{-}} \mathbf{K} \operatorname{grad} u \cdot \operatorname{grad} u d x d \tau-\int_{0}^{t}\left\langle u^{-}, \phi\right\rangle d \tau=0 .
$$

We conclude that $u \equiv 0$ in $\Omega_{T}^{-}$and $u \equiv 0$ in $\Omega_{T}^{+}$; hence $\varphi \equiv 0$, and by (3.10), $C=0$.

Lemma 4.4 states that there is a bounded linear map $\mathscr{S}_{0}: P_{T} \rightarrow Q_{T}^{+}$with $\mathscr{S}_{0}\{F, G, A\}$ the solution $\{u, \varphi, c\}$ of (4.4). Now consider the map $J: Q_{T}^{+} \rightarrow P_{T}$ defined by

$$
J\{u, \varphi, C\}=\{\mathscr{R} u, \mathscr{J} u, 0\},
$$

where $\mathscr{R}$ and $\mathscr{J}$ are defined in (4.2).

Lemma 4.5. $J$ is a compact map from $Q_{T}^{+}$into $P_{T}$.

Proof. Let $\left\{u_{n}, \varphi_{n}, C_{n}\right\}$ be a bounded sequence in $Q_{T}^{+}$. Then $\left\{u_{n}\right\}$ is bounded in $H_{T}$, and $\dot{u}_{n}$ is bounded in $H_{T}^{\prime}$. This implies ([6]) that $\left\{u_{n}\right\}$ is bounded in $H_{1 / 2}(0, T$ : $\left.\left[H, H^{\prime}\right]_{1 / 2}\right)=H_{1 / 2}\left(0, T: L_{2}(\Omega)\right)$. Now the injection of $H_{\gamma}(0, T: \mathbf{X})$ into $L_{2}(0, T: \mathbf{Y})$ for any $\gamma>0$ is compact whenever the embedding of $\mathbf{X}$ into $\mathbf{Y}$ is compact. Taking $\mathbf{X}=L_{2}(\Omega)$ and $\mathbf{Y}=H^{\prime}$ we see that the injection of $H_{1 / 2}\left(0, T: L_{2}(\Omega)\right)$ into $H_{T}^{\prime}$ is compact. Hence there is a subsequence $\left\{u_{n}\right\}$ which converges in $H_{T}^{\prime}$. 
Next we see that since $\left\{u_{n}\right\}$ is bounded in both $H_{T}$ and $H_{1 / 2}\left(0, T: L_{2}(\Omega)\right)$, we have $u_{n}^{-} \in H_{1 / 4}\left(0, T: L_{2}(\Gamma)\right.$ ) (see [6]). But by Lemma 3.1, $D$ is a bounded map from $H_{r}$ into $H_{r+2}$; hence $\left\{D u_{n}\right\}$ is bounded in $H_{1 / 4}\left(0, T: H_{2}(\Gamma)\right)$. The embedding of $H_{2}(\Gamma)$ into $H_{1 / 2}(\Gamma)$ is compact so that, as above, $H_{1 / 4}\left(0, T: H_{2}(\Gamma)\right)$ is compactly embedded in $L_{2}\left(0, T: H_{1 / 2}(\Gamma)\right)$. Hence $\mathscr{J} u_{n}=D u_{n}$ has a convergent subsequence in $B_{T}^{\prime}$. This completes the proof.

We see that $\{u, \varphi, C\}$ is a solution of (3.4) if and only if it satisfies

$$
\{u, \varphi, C\}=\mathscr{S}_{0}\{F, G, A\}+\mathscr{S}_{0} J\{u, \varphi, C\} .
$$

Now $\mathscr{S}$ is a bounded map from $P_{T}$ to $Q_{T}^{+}$, and by Lemma $4.5, J$ is compact from $Q_{T}^{+}$to $P_{T}$. Hence $\mathscr{S J}$ is compact from $Q_{T}^{+}$to itself, and (4.23) is a Riesz-Schauder system. Uniqueness for (VP) shows that the only solution of the homogeneous equation for (4.23) is $\{u, \varphi, C\}=\{0,0,0\}$. Hence (4.20) has a unique solution and so does (VP).

Proof of Lemma 4.1. It is known [4] that $-S$ is coercive, that is,

$$
-\langle S[\Phi], \Phi\rangle \geqslant \gamma^{\prime}\|\Phi\|_{B}^{2},
$$

provided that the diameter of $\Gamma$ is sufficiently small. Assume the origin is inside $\Gamma$. For a $\varphi$ with $m[\varphi]=0$, we have for any $\delta$,

$$
S[\varphi](x)=\frac{1}{2 \pi} \int_{\Gamma} \varphi(y) \log |x-y| d s_{y}=\frac{1}{2 \pi} \int_{\Gamma} \varphi(y) \log \frac{|x-y|}{\delta} d s_{y} .
$$

Put $x=\delta \xi, y=\delta \eta, \varphi(\delta \eta)=\Phi(\eta)$, and $\Gamma^{\prime}=\Gamma / \delta$. Choose $\delta$ so that the diameter of $\Gamma^{\prime}$ is small enough for (4.24) to hold. Then $S[\varphi](x)=\delta S_{\Gamma^{\prime}} \Phi(\delta \xi)$, where $S_{\Gamma^{\prime}}$ is the simple layer for $\Gamma^{\prime}$. Eq. (4.24) yields

$$
-\frac{1}{2 \pi} \int_{\Gamma^{\prime}} \Phi(\xi) S_{\Gamma^{\prime}} \Phi(\xi) d s_{\xi} \geqslant \gamma^{\prime}\|\Phi(\xi)\|_{B}^{2},
$$

and reversing the transformation yields

$$
-\langle S \varphi, \varphi\rangle \geqslant \gamma^{\prime} \delta\|\varphi\|_{-1 / 2}^{2}
$$

which is (4.7).

The proof of 'Theorem 3.2 is now complete.

5. Proof of optimality. We follow the ideas of [3]. We require the following definitions.

Definition 5.1. The Galerkin operator $G^{h}$ for (VP) is the map on $Q_{T}^{+}$defined by $G^{h}\{u, \varphi, \psi\}=\{\tilde{u}, \tilde{\varphi}, \tilde{C}\}$, where $\tilde{u}(x, 0)=0$ and for any $\left\{v^{h}, \psi^{h}, k\right\}$,

$$
\left\langle\dot{\tilde{u}}, v^{h}\right\rangle+a\left(\{\tilde{u}, \tilde{\varphi}, \tilde{C}\},\left\{v^{h}, \psi^{h}, k\right\}\right)=\left\langle\dot{u}, v^{h}\right\rangle+a\left(\{u, \varphi, C\},\left\{v^{h}, \psi^{h}, k\right\}\right) .
$$

DEFinition 5.2. The Galerkin operator $G_{0}^{h}$ for (4.5) is the map on $Q_{T}^{+}$defined by $G_{0}^{h}\{u, \varphi, \psi\}=\{\tilde{u}, \tilde{\psi}, \tilde{C}\}$, where $\tilde{u}^{h}(x, 0)=0$ and for any $\left\{v^{h}, \psi^{h}, k\right\}$,

$$
\left\langle\dot{\tilde{u}}, v^{h}\right\rangle+a_{0}\left(\{\tilde{u}, \tilde{\varphi}, \tilde{C}\},\left\{v^{h}, \psi^{h}, k\right\}\right)=\left\langle\dot{u}, v^{h}\right\rangle+a_{0}\left(\{u, \varphi, C\},\left\{v^{h}, \psi^{h}, k\right\}\right) .
$$

LEMMA 5.1. $G_{0}^{h}$ is well defined and for any $\{u, \varphi, C\} \in Q_{T}^{+}$

$$
\left\|\{u, \varphi, C\}-G_{0}^{h}\{u, \varphi, C\}\right\|_{Q_{T}^{+}} \rightarrow 0 \text { as } h \rightarrow 0 .
$$


Proof. It follows from Lemma 4.2 that $G_{0}^{h}$ is well defined. The stability result (4.15) can be used to show convergence of $G_{0}^{h}\{u, \varphi, C\}$ to $\{u, \varphi, C\}$ as follows. Let $\left\{w^{h}, \chi^{h}, r^{h}\right\}$ be an arbitrary element of $Q_{T}^{+}$and put

$$
\begin{gathered}
\left\{e_{1}, e_{2}, e_{3}\right\}=G_{0}\{u, \varphi, C\}-\left\{w^{h}, \chi^{h}, r^{h}\right\}, \\
\left\{\varepsilon_{1}, \varepsilon_{2}, \varepsilon_{3}\right\}=\{u, \varphi, C\}-\left\{w^{h}, \chi^{h}, r^{h}\right\} .
\end{gathered}
$$

Then (5.12) yields

$$
\left\langle\dot{e}_{1}, v^{h}\right\rangle+a_{0}\left(\left\{e_{1}, e_{2}, e_{3}\right\},\left\{v^{h}, \chi^{h}, k\right\}\right)=\left\langle\dot{\varepsilon}_{1}, v^{h}\right\rangle+a_{0}\left(\left\{\varepsilon_{1}, \varepsilon_{2}, \varepsilon_{3}\right\},\left\{v^{h}, \psi^{h}, k\right\}\right) .
$$

The right side of (5.3) can be written in the same form as in (4.5), and the estimate (4.15) can be applied to yield

$$
\left\|\left\{e_{1}, e_{2}, e_{3}\right\}\right\|_{Q_{T}^{+}} \leqslant K^{\prime}\left\|\left\{\varepsilon_{1}, \varepsilon_{2}, \varepsilon_{2}\right\}\right\|_{Q_{T}^{+}} .
$$

Thus we obtain

$$
\left\|G_{0}\{u, \varphi, c\}-\{u, \varphi, c\}\right\|_{Q_{T}^{+}} \leqslant K \inf _{\left\{w^{h}, \chi^{h}, r^{h}\right\} \in Q_{T}^{h}}\left\|\{u, \varphi, c\}-\left\{w^{h}, \chi^{h}, r^{h}\right\}\right\|_{Q_{T}^{+}} \cdot(5 .
$$

Equation (5.4) and our assumption A.2 yield the assertion of the lemma.

LEMma 5.2. $\left\|G_{0}^{h}\right\|$ is bounded independently of $h$.

Proof. Put $\left\{w^{h}, \chi^{h}, r^{h}\right\}=\{0,0,0\}$ in (5.4) to conclude that $G_{0}^{h}-I$ is uniformly bounded and hence so is $G_{0}^{h}$.

Let $\mathscr{S}: P_{T} \rightarrow Q_{T}^{+}$be the solution operator for (VP). We know this exists and is bounded from Sec. 4 .

Lemma 5.3. Let $J$ be defined by (4.22). Then

$$
\left\|\mathscr{S} J\left(I-G_{0}^{h}\right)\right\|_{B L\left(Q_{T}^{+} \cdot Q_{T}^{+}\right)} \rightarrow 0 \quad \text { as } h \rightarrow 0 .
$$

Proof. We note that $J\left(I-G_{0}^{h}\right)$ is an operator from $Q_{T}^{+}$into $P_{T}$ and $\mathscr{S}$ is a bounded operator from $P_{T}$ into $Q_{T}^{+}$. Suppose (5.5) does not hold. Then we can find $\varepsilon>0$, a sequence $h_{n} \downarrow 0$, and $\left\{v_{n}, \varphi_{n}, C_{n}\right\} \in Q_{T}^{+}$with $\left\|\left\{v_{n}, \varphi_{n}, C_{n}\right\}\right\|_{Q_{T}^{+}}=1$ such that $\| \mathscr{S} J(I-$ $\left.G_{0}^{h}\right)\left\{v_{n}, \varphi_{n}, C_{n}\right\} \|_{Q_{T}^{+}} \geqslant \varepsilon$. Since $\left\|I-G_{0}^{h_{n}}\right\|$ is uniformly bounded, $\left(I-G_{0}^{h_{n}}\right)\left\{v_{n}, \varphi_{n}, C_{n}\right\}=$ $\left\{w_{n}, \chi_{n}, r_{n}\right\}$ is a bounded sequence in $Q_{T}^{+}$and, by construction,

$$
\left\langle\dot{w}_{n}, v^{h_{n}}\right\rangle+a_{0}\left(\left\{w_{n}, \chi_{n}, r_{n}\right\},\left\{v^{h_{n}}, \psi^{h_{n}}, k\right\}\right)=0
$$

for any $\left\{v^{h_{n}}, \psi^{h_{n}}, k\right\} \in \mathscr{H}^{h_{n}}$. Since the sequence $\left\{w_{n}, \chi_{n}, r_{n}\right\}$ is bounded, it has a subsequence converging weakly to $\{w, \chi, r\}$ and from (5.6) and A.2 one concludes that $\{w, \chi, r\}$ is a solution of (4.5) for $\{F, G, A\}=\{0,0,0\}$. Hence $\{w, \chi, r\}=\{0,0,0\}$. But the map $J$ is compact, so that $J\left\{w_{n}, \chi_{n}, r_{n}\right\}$ converges strongly to zero and hence so does $\mathscr{S} J\left\{w_{n}, \chi_{n}, r_{n}\right\}$, which gives us a contradiction.

By Lemma (5.3), we see that the operator $\tilde{G}^{h}=G_{0}^{h}\left[I-\mathscr{S} J\left(I-G_{0}^{h}\right)\right]^{-1}$ exists for $h$ sufficiently small. 
LEMma 5.4. $\tilde{G}^{h}=G^{h}$.

Proof. Put $Z=(z, \xi, p)=\left(I-\mathscr{S} J\left(I-G_{0}^{h}\right)\right)^{-1} U$ and $U=\{u, \varphi, c\}$. Then $Z-\mathscr{S} J(I$ $\left.-G_{0}^{h}\right) Z=U$ and, in obvious notation,

$$
\begin{aligned}
\langle\dot{z}, v\rangle+a(\{z, \xi, p\},\{v, \psi, k\})-\mathscr{F}_{J\left(I-G_{0}^{h}\right) Z}(\{v, \psi, k\}) \\
=\langle\dot{u}, v\rangle+a(\{u, \varphi, C\},\{v, \psi, k\}) .
\end{aligned}
$$

If we use the definition of $J$ in (4.22), we see that (5.7) is the same as

$$
\begin{aligned}
\langle\dot{z}, v\rangle+a_{0}(\{z, \xi, p\},\{v, \psi, k\})+\mathscr{F}_{J G_{0}^{h} Z}(\{v, \psi, k\}) \\
=\langle\dot{u}, v\rangle+a(\{u, \varphi, C\},\{v, \psi, k\}) .
\end{aligned}
$$

But we have, again by (4.22),

$$
\mathscr{F}_{J G_{0}^{h} Z}(\{v, \psi, k\})=a\left(G_{0}^{h} Z,\{v, \psi, k\}\right)-a_{0}\left(G_{0}^{h} Z,\{v, \psi, k\}\right) .
$$

Hence (5.8) can be written

$$
\begin{aligned}
a\left(G_{0}^{h} Z,\{v, \psi, k\}\right)+\langle\dot{z}, v\rangle & -a_{0}\left(Z-G_{0}^{h} Z,\{v, \psi, k\}\right) \\
& =\langle\dot{u}, v\rangle+a(\{u, \varphi, C\},\{v, \psi, k\}) .
\end{aligned}
$$

Now set $\tilde{Z}=G_{0}^{h} Z=\{\tilde{z}, \tilde{\xi}, \tilde{p}\}$. Then,

$$
\left\langle\dot{\tilde{z}}, v^{h}\right\rangle+a_{0}\left(\tilde{Z},\left\{v^{h}, \psi^{h}, k\right\}\right)=\left\langle\dot{z}, v^{h}\right\rangle+a_{0}\left(Z,\left\{v^{h}, \psi^{h}, k\right\}\right) .
$$

If we combine (5.10) with (5.9) for $\{v, \psi, k\}=\left\{v^{h}, \psi^{h}, k\right\}$, we obtain finally

$$
\left\langle\dot{\tilde{z}}, v^{h}\right\rangle+a\left(\{\tilde{z}, \tilde{\xi}, \tilde{p}\},\left\{v^{h}, \psi^{h}, k\right\}\right)=\left\langle\dot{u}, v^{h}\right\rangle+a\left(\{u, \varphi, C\},\left\{v^{h}, \psi^{h}, k\right\}\right),
$$

which proves the lemma.

We can now complete the proof of Theorem 3.3. By our construction, we see that $G^{h}$ is uniformly bounded in the operator norm. Now for the solution of (VP), we have $G^{h}\{w, \varphi, C\}=\left\{u^{h}, \varphi^{h}, C^{h}\right\}$, the solution of $(\mathrm{AVP})_{h}$. For any $\left\{w^{h}, \chi^{h}, l^{h}\right\}$ we have $G^{h}\left\{w^{h}, \chi^{h}, l^{h}\right\}=\left\{w^{h}, \chi^{h}, l^{h}\right\}$. Hence,

$$
\begin{aligned}
\|\{u, \varphi, C\}- & \left\{u^{h}, \varphi^{h}, C^{h}\right\} \|_{Q_{+}^{+}} \\
& \leqslant\left\|\{u, \varphi, C\}-G^{h}\{u, \varphi, C\}+G^{h}\left\{w^{h}, \chi^{h}, l^{h}\right\}-\left\{w^{h}, \chi^{h}, l^{h}\right\}\right\|_{Q_{\dot{T}}} \\
& \leqslant\left\|\{u, \varphi, C\}-\left\{w^{h}, \chi^{h}, l^{h}\right\}\right\|_{Q_{\dot{T}}}+\left\|G^{h}(\{u, \varphi, C\})-\left\{w^{h}, \chi^{h}, l^{h}\right\}\right\|_{Q_{T}^{+}},
\end{aligned}
$$

from which (3.14) follows.

6. Numerical considerations. In this section, we discuss approximate subspaces for which assumptions A.1 and A.2 are satisfied and show that quasioptimal convergence is obtained for such spaces.

We introduce polygonal grids $\Delta_{\Omega}$ and $\Delta_{\Gamma}$ on $\Omega$ and $\Gamma$, respectively, with generic mesh spacings $h_{\Omega}$ and $h_{\Gamma}$. Let $M^{h} \subset H^{\prime}(\Omega)$ be a space of piecewise polynomials of degree $\leqslant k-1$ on $\Delta_{\Omega}$ and $B^{h} \subset H_{-1 / 2}(\Gamma)$ a space of piecewise polynomials of degree $\leqslant l-1$ on $\Delta_{\Gamma}$. We identify the parameter $h$ with $\max \left(h_{\Omega}, h_{\Gamma}\right)$. With the above definitions of $H^{h}$ and $B^{h}$, we can show that the $Q_{T}^{h}=H_{T}^{h} \times B_{T}^{h} \times \mathbf{R}_{T}$ approximate $Q_{T}^{+}$in the sense of A.1. 
We consider the case of $k=2$ and $l=1$, that is, when piecewise linears are used on $\Omega$ and piecewise constants on $\Gamma$. Let

$$
\begin{gathered}
Z=\left\{(v, \psi, C) \mid v \in L^{2}\left(0, T: H_{2}(\Omega)\right), \dot{v} \in L^{2}\left(0, T: H_{1}(\Omega)\right),\right. \\
\left.v(0)=0, C \in \mathbf{R}_{T}, \psi \in L^{2}\left(0, T: H_{1 / 2}(\Gamma)\right)\right\} .
\end{gathered}
$$

Then it is known [2] that, given $v \in H_{2}(\Omega)$ and $\psi \in H_{1 / 2}(\Gamma)$.

$$
\begin{gathered}
\inf _{w^{h} \in H^{h}}\left\|v-w^{h}\right\|_{1, \Omega} \leqslant K h\|v\|_{2, \Omega}, \\
\inf _{x^{h} \in B^{h}}\left\|\psi-x^{h}\right\|_{-1 / 2, \Gamma} \leqslant K h\|\psi\|_{1 / 2, \Gamma}
\end{gathered}
$$

for some constant $0<K<\infty$ independent of $h$. Hence, given $V=\left(v, \psi_{0}, C\right) \in Z$, we can integrate (6.1) and (6.2) in time to obtain

$$
\inf _{W^{h} \in Q_{T}^{h}}\left\|V-W^{h}\right\|_{Q_{T}} \leqslant K^{\prime} h,
$$

where $K^{\prime}$ depends upon $V$ but not $h$. Moreover, we can simultaneously approximate $v$ and $\dot{v}$ by a function $w^{h}(t) \in H_{T}^{h}$ and its derivative such that for almost all $t$, we have (6.1) together with

$$
\left\|\dot{v}(t)-\dot{w}^{h}(t)\right\|_{0} \leqslant K h\|\dot{v}(t)\|_{1, \Omega} .
$$

Integrating (6.4) with respect to $t$ and combining it with (6.3), we obtain for $V \in Z$,

$$
\inf \left\|V-W^{h}\right\|_{Q_{T}^{+}} \leqslant C h .
$$

A.1 is then a consequence of the following lemma.

Lemma 6.1. If $Z$ is a dense subspace of $Q_{T}^{+}$such that (6.5) holds, then $\inf _{V^{h} \in Q_{T}^{h}}\left\|V-V^{h}\right\|$ $\rightarrow 0$ for all $V \in Q_{T}^{+}$as $h \rightarrow 0$.

Proof. Let $V \in Q^{+} / Z$. Since $Z$ is dense in $Q_{T}^{+}$, there exists a sequence $\left\{V^{n}\right\} \in Z$ such that $\left\|V^{n}-V\right\|_{Q_{T}^{+}} \rightarrow 0$. Now

$$
\inf \left\|V-V^{h}\right\|_{Q_{T}^{+}} \leqslant\left\|V-V^{n}\right\|_{Q_{T}^{+}}+\inf \left\|V^{n}-V^{h}\right\|_{Q_{T}^{+}} .
$$

The right side can be made arbitrarily small by choosing $n$ sufficiently large and then with $n$ fixed, letting $h \rightarrow 0$.

In order for A.2 to be satisfied, a sufficient condition is that $H^{h}$ satisfy the following inverse property, which will hold for any grid $\Delta_{\Omega}$ that is regular.

Definition 6.1. $H^{h}$ is said to have the inverse property if there is a number $0<C_{I}<\infty$ such that

$$
\left\|v^{h}\right\|_{1} \leqslant C_{I} h_{\Omega}^{-1}\left\|v^{h}\right\|_{0} \quad \text { for all } v^{h} \in H^{h} .
$$

Let $v \in H^{\prime}(\Omega)$ and $P^{h} v$ be its $L^{2}$ projection in $H^{h}$, as defined in A.1. Then for any $w^{h} \in H^{h}$,

$$
\left\|P^{h} v-w^{h}\right\|_{0} \leqslant\left\|v-w^{h}\right\|_{0} .
$$

Also,

$$
\begin{aligned}
\left\|P^{h} v\right\|_{1} & \leqslant\|v\|_{1}+\left\|v-w^{h}\right\|_{1}+\left\|P^{h} v-w^{h}\right\|_{1} \\
& \leqslant\|v\|_{1}+\left\|v-w^{h}\right\|_{1}+C_{I} h_{\Omega}^{-1}\left\|v-w^{h}\right\|_{0} \\
& \leqslant C\|v\|_{1}
\end{aligned}
$$


since

$$
\inf _{w^{h} \in H^{h}}\left(\left\|v-w^{h}\right\|_{1}+C_{l} h_{\Omega}^{-1}\left\|v-w^{h}\right\|_{0}\right) \leqslant K\|v\|_{1}
$$

(see [2]). This proves A.2.

Hence, we see that (6.5) combined with (3.14) yields $O(h)$ convergence in $Q_{T}^{+}$, that is,

$$
\left\|V-V^{h}\right\|_{Q_{T}^{+}} \leqslant C h
$$

provided that the true solution $U \in Z$.

Note that unlike the usual condition obtained when Lagrange multipliers are used for the Poisson equation, we do not require a restriction of the form $h_{\Omega} / h_{\Gamma} \rightarrow 0$ as $h_{\Omega}$, $h_{\Gamma} \rightarrow 0$. In particular, this implies that $\Delta_{\Gamma}$ can be taken to be the grid introduced on the boundary by $\Delta_{\Omega}$.

7. Physical considerations. The problem we want to consider is that in which we have an initial electromagnetic field in all space, which we think of as air, a dielectric. Metallic obstacles are introduced and the problem is to determine the subsequent field. We term this the eddy current problem.

Electromagnetic fields are governed by Maxwell's equations,

$$
\operatorname{curl} \mathscr{E}=-\mathscr{B}_{t}, \quad \operatorname{curl} \mathscr{H}=\mathscr{I},
$$

where $\mathscr{E}$ and $\mathscr{H}$ are electric and magnetic fields, $\mathscr{B}$ is magnetic displacement, and $\mathscr{I}$ is current density. For a homogeneous isotropic dielectric, such as air, it is customary to neglect conduction current and use the constitutive relations

$$
\mathscr{H}=\mu_{0}^{-1} \mathscr{B}, \quad \mathscr{I}=\varepsilon_{0} \mathscr{E}_{t},
$$

$\mu_{0}$ and $\varepsilon_{0}$ constants. For a nonferromagnetic metal which is electrically isotropic but magnetically anisotropic and inhomogeneous, one usually neglects displacement current and assumes

$$
\mathscr{H}=\mathscr{H}^{\prime} \mathscr{B}, \quad \mathscr{I}=\sigma E
$$

where the scalar $\sigma$ and the symmetric positive definite transformation $\mathscr{K}^{\prime}$ depend on position.

We suppose that we have an incident field $\mathscr{E}^{0}, \mathscr{H}^{0}, \mathscr{B}^{0}$ satisfying (7.1) and (7.2) in all space. Then the distorted field satisfies (7.1) and (7.3) in the obstacles and (7.1) and (7.2) outside. Across the interface the tangential components of both $\mathscr{E}$ and $\mathscr{H}$ must be continuous.

We scale the problem. Let $L$ and $T$ be a representative length and time and replace $x$ and $t$ by $x / L$ and $t / T$. Let $\mathscr{B}_{0}$ be a representative magnitude for $\mathscr{B}_{0}$ and put

$$
\mathscr{B}=\mathscr{B}_{0} \boldsymbol{B}, \quad \mathscr{E}=(L / T) \mathscr{B}_{0} \boldsymbol{E}, \quad \mathscr{K}^{\prime}=\mu_{0}^{-1} \boldsymbol{K}^{\prime},
$$

so that $\boldsymbol{B}, \boldsymbol{E}$, and $\boldsymbol{K}^{\prime}$ are dimensionless. Then

$$
\begin{array}{ll}
\operatorname{curl} \boldsymbol{E}=-\boldsymbol{B}_{t}, & \operatorname{curl} \boldsymbol{B}=\beta^{2} \boldsymbol{E}_{t} \quad \text { in air, } \\
\operatorname{curl} \boldsymbol{E}=-B_{t}, & \operatorname{curl} \boldsymbol{K}^{\prime} \boldsymbol{B}=\alpha \boldsymbol{E} \quad \text { in metal, }
\end{array}
$$

where

$$
\beta^{2}=\mu_{0} \varepsilon_{0} L^{2} / T^{2}, \quad \alpha=\mu_{0} \sigma L^{2} / T
$$


are also dimensionless. We let $\boldsymbol{E}$ and $\boldsymbol{B}$ denote the scattered field, total minus incident, outside. Then the interface conditions are

$$
\boldsymbol{E}_{\text {tang }}^{-}=\boldsymbol{E}_{\text {tang }}^{+}+\boldsymbol{E}_{\text {tang }}^{0}, \quad\left(\boldsymbol{K}^{\prime} \boldsymbol{B}\right)_{\text {tang }}^{-}=\boldsymbol{B}_{\text {tang }}^{+}+\boldsymbol{B}_{\text {tang }}^{0}
$$

on the interface, where + and - denote limits from air and metal.

We now specialize the geometry. Assume the metallic obstacle is a uniform cylinder of cross-section $\Omega$ parallel to the $z$-axis with $\boldsymbol{K}^{\prime}$ and $\alpha$ depending only on $x$ and $y$ and with $K_{i j}^{\prime}=0$ if $i$ or $j=3$. Assume that all fields are transverse magnetic, that is,

$$
\boldsymbol{E}=E(x, y, t) \hat{k}, \quad \boldsymbol{B}=B^{1}(x, y, t) \hat{i}+B^{2}(x, y, t) \hat{j} .
$$

One can verify that such fields will satisfy the Maxwell equations if

$$
\begin{gathered}
B^{1}(x, y, t)=u_{y}(x, y, t), \quad B^{2}(x, y, t)=-u_{x}(x, y, t), \\
E(x, y, t)=-u_{t}(x, y, t)
\end{gathered}
$$

with

$$
\begin{aligned}
\Delta u=u_{x x}+u_{y y}=\beta^{2} u_{t t} & \text { in air, } \\
\operatorname{div}(K \operatorname{grad} u)=\alpha u_{t} & \text { in metal, }
\end{aligned}
$$

where

$$
\boldsymbol{K}^{\prime}=\left(\begin{array}{ll}
K_{11}^{\prime} & K_{12}^{\prime} \\
K_{21}^{\prime} & K_{22}^{\prime}
\end{array}\right), \quad \boldsymbol{K}=\left(\begin{array}{cc}
K_{22}^{\prime} & -K_{21}^{\prime} \\
-K_{12}^{\prime} & K_{11}^{\prime}
\end{array}\right)
$$

so that $K$ is still symmetric and positive definite. If the incident field is generated by $u^{0}$, then the interface conditions (7.7) yield

$$
u^{-}=u^{+}+u^{0}, \quad(K \operatorname{grad} u)^{-} \cdot \boldsymbol{n}=(\operatorname{grad} u)^{+} \cdot \boldsymbol{n}+\operatorname{grad} u^{0} \cdot \boldsymbol{n} \quad \text { on } \Gamma .
$$

The above simplifications yield a $u$ satisfying $(1.4)_{1}$ and $(1.4)_{3}$ with

$$
f=u^{0}, \quad g=\operatorname{grad} u^{0} \cdot \boldsymbol{n}=u_{n}^{0} \quad \text { on } \Gamma .
$$

We assume all fields start from rest, and then we obtain $(1.4)_{2}$ and have the problem (PHP).

For fields that do not vary too rapidly with time, the parameter $\alpha$ is usually $O(1)$ while $\beta^{2} \ll 1$. Hence a natural approximation is to set $\beta^{2}=0$, in which case we no longer need the initial conditions in $\Omega^{+}$. We do, however, have to impose a growth condition as $|x| \rightarrow \infty$. We require that the total fields remain bounded at infinity. There is a technical difficulty here. If we require that the incident field generator $u^{0}$ stay bounded at infinity and be defined everywhere, it would have to be a constant, since $\Delta u_{0}=0$. Then (PEP) is trivial. To obtain a meaningful problem, we give up the requirement that $u_{0}$ be everywhere defined and bounded. We have in mind the case where $\boldsymbol{E}^{0}$ and $\boldsymbol{B}^{0}$ are generated by wires parallel to the cylinder and carrying current. For such a wire at $x^{0} \in \Omega^{+}$carrying current $I(t)$, the corresponding $u_{0}$ in the $\beta=0$ limit is

$$
u^{0}(x, t)=(1 / 2 \pi) I(t) \log \left|x-x_{0}\right| .
$$

If we use such a $u^{0}$, we observe that to keep the total electric field bounded at infinity we must allow the scattered field generator $u$ to grow logarithmically at infinity to compensate for (7.12). This is the origin of $(1.3)_{4}$. 
If we assume $u^{0}$ is defined and satisfies $\Delta u^{0}=0$ everywhere except at a finite number of points $x_{0}$, then in particular we will have $\Delta u_{0}=0$ in $\Omega^{-}$; hence

$$
\int_{\Gamma} g d s=\int_{\Gamma} u_{n}^{0} d s=0
$$

which is (1.5).

\section{REFERENCES}

[1] J. Bielak and R. C. MacCamy, An exterior interface problem in two-dimensional elastodynamics, Quart. Appl. Math. 41, 143-159 (1983)

[2] P. Ciarlet, The finite element method for elliptic problems, North-Holland (1983)

[3] S. Hildebrandt and E. Weinholz, Constructive proofs of representation theorems in separable Hilbert spaces, Comm. Pure Appl. Math. 17, 369-373 (1964)

[4] G. C. Hsiao, and W. L. Wendland, A finite element method for some integral equations of the first kind, J. Math. Anal. Appl. 58, 449-481 (1977)

[5] C. Johnson and J. C. Nedelec, On the coupling of boundary integral and finite element methods, Math. Comp. 35, 1063-1079 (1980)

[6] J. L. Lions and E. Magenes, Non-homogeneous boundary value problems and applications, Springer-Verlag, New York (1972)

[7] M. Suri, Mixed variational principles for time dependent problems, Thesis, Dept. of Mathematics, CarnegieMellon University (August 1983) 